\title{
Trends in cancer mortality among people with vs without diabetes in the USA, 1988-2015
}

\author{
Jessica L. Harding ${ }^{1} \cdot$ Linda J. Andes ${ }^{1} \cdot$ Edward W. Gregg ${ }^{1} \cdot$ Yiling J. Cheng $^{1} \cdot$ Hannah K. Weir $^{2} \cdot$ Kai M. Bullard ${ }^{1}$. \\ Nilka Ríos Burrows ${ }^{1} \cdot$ Giuseppina Imperatore ${ }^{1}$
}

Received: 6 June 2019 / Accepted: 6 August 2019/Published online: 12 September 2019

(C) Springer-Verlag GmbH Germany, part of Springer Nature 2019

\begin{abstract}
Aims/hypothesis Cancer-related death is higher among people with vs without diabetes. However, it is not known if this excess risk has changed over time or what types of cancer may be driving these changes.

Methods To estimate rates of site-specific cancer mortality in adults with vs without self-reported diagnosed diabetes, we used data from adults aged $\geq 18$ years at the time of the interview who participated in the 1985-2012 National Health Interview Survey. Participants' data were linked to the National Death Index by the National Center for Health Statistics to determine vital status and cause of death through to the end of 2015. Cancer deaths were classified according to underlying cause of death. Death rates for five time periods (1988-1994, 1995-1999, 2000-2004, 2005-2009, 2010-2015) were estimated using discrete Poisson regression models adjusted for age, sex and race/ethnicity with $p$ for linear trend reported $\left(p_{\text {trend }}\right)$. Site-specific cancer mortality rates were stratified by diabetes status and period, and total cancer mortality rates were additionally stratified by sex, race/ethnicity, education and BMI status. Results Among adults with diabetes, age-adjusted cancer mortality rates (per 10,000 person-years) declined $25.5 \%$ from 39.1 (95\% CI 30.1, 50.8) in 1988-1994 to $29.7(26.6,33.1)$ in 2010-2015, $p_{\text {trend }}<0.001$. Among adults without diabetes, rates declined $25.2 \%$ from $30.9(28.6,33.4)$ in $1988-1994$ to $23.2(22.1,24.2)$ in $2010-2015, p_{\text {trend }}<0.01$. Adults with diabetes remained approximately $30 \%$ more likely to die from cancer than people without diabetes, and this excess risk did not improve over time. In adults with diabetes, cancer mortality rates did not decline in some population subgroups (including black people, people with lower levels of education and obese people), and the excess risk increased for obese adults with vs without diabetes. Declines in total cancer mortality rates in adults with diabetes appear to be driven by large relative declines in cancers of the pancreas $(55 \%)$ and breast $(65 \%)$, while for lung cancer, declines are modest $(7 \%)$.

Conclusions/interpretation Declines in cancer mortality rates were observed in adults with and without diabetes. However, adults with diabetes continue to be more likely to die from cancer than people without diabetes. This study highlights the continued need for greater cancer risk-factor mitigation, especially in adults with diabetes.
\end{abstract}

Keywords Cancer $\cdot$ Diabetes $\cdot$ Epidemiology $\cdot$ Mortality $\cdot$ Trends

Electronic supplementary material The online version of this article (https://doi.org/10.1007/s00125-019-04991-x) contains peer-reviewed but unedited supplementary material, which is available to authorised users.

Jessica L. Harding jharding@cdc.gov

1 Division of Diabetes Translation, Centers for Disease Control and Prevention, 4770 Buford Hwy NE, Mailstop F75,

Atlanta, GA 30341, USA

2 Division of Cancer Prevention and Control, Centers for Disease Control and Prevention, Atlanta, GA, USA

\author{
Abbreviations \\ NCHS National Center for Health Statistics \\ NDI National Death Index \\ NHIS National Health Interview Survey
}

\section{Introduction}

Diabetes is associated with an approximately $75 \%$ increase in mortality risk in the US population and the typical adult diagnosed with diabetes at age 60 loses 5 years of their life to the disease $[1,2]$. Cardiovascular diseases have historically accounted for the largest number of those deaths. However, 


\section{Research in context}

\section{What is already known about this subject?}

- It is well known that people with diabetes have an increased risk for death from several cancer types, compared with people without diabetes

- It is not known if this excess risk has changed over time or what types of cancer may be driving these changes

\section{What is the key question?}

- Has the excess risk of site-specific cancer mortality in people with vs without diabetes changed in the USA between 1988 and 2015?

\section{What are the new findings?}

- Between 1988 and 2015, adults both with and without diabetes experienced a decline in cancer mortality rates. However, during the 2010-2015 time period, adults with diabetes remained approximately $30 \%$ more likely to die from cancer than people without diabetes, and this excess risk did not improve over time

- Cancer mortality rates did not decline in some of the population subgroups of people with diabetes (including black people, people with lower levels of education and obese people), and the excess risk increased for obese adults with vs without diabetes

- Declines in total cancer mortality rates in adults with diabetes appear to be driven by large relative declines in cancers of the pancreas and breast, while for lung cancer declines are modest

\section{How might this impact on clinical practice in the foreseeable future?}

- This study highlights the continued need for greater cancer risk-factor mitigation in adults with diabetes to prevent premature death from cancer

some population studies have suggested there may be shifts underway in the epidemiology of diabetes-related complications and deaths $[3,4]$. In the USA, rates of vascular disease mortality have declined more than other types of morbidities leading to a change in proportional mortality [5].

Rates of cancer-related deaths among the diabetic population could be affected by a decreasing competing risk of deaths from vascular disease. However, diabetes has been independently associated with the incidence of several types of cancer, particularly of the liver, breast, endometrium, pancreas, colon and kidney [6], as well as with cancer-related mortality [7, 8]. In addition, insulin treatment and oral glucose-lowering medications have been associated with increased cancer risk, although the causal association of this connection remains unclear $[9,10]$.

Despite the growing awareness and apparent complexity of the association of diabetes with cancer, no nationally representative studies in the USA have examined trends in cancerrelated mortality rates among adults with diabetes. Therefore, to address this knowledge gap, we used nationally representative data from the USA to estimate trends in mortality rates of common cancer types among adults with and without selfreported diagnosed diabetes between 1988 and 2015 .

\section{Methods}

Data sources Data came from the 1985-2012 National Health Interview Surveys (NHIS) data linked to the National Death
Index (NDI) by the National Center for Health Statistics (NCHS) data linkage program [11]. The NHIS is a nationally representative household survey of the civilian noninstitutionalised US population. The NHIS sample is drawn from each state and the District of Columbia using a multistage probability design to select an average of 35,000 households containing approximately 87,500 persons per year [12]. NHIS data are used to monitor the health of the nation, the prevalence and incidence of disease, the extent of disability and the use of healthcare services. Self-reported data on diagnosed diabetes status, age, sex, race/ethnicity (non-Hispanic white, non-Hispanic black, Hispanic, or other), education (below high school, high school, above high school), family income (defined as income poverty ratio $<2.0$ [low income], 2.0-4.9 [moderate income], $\geq 5.0$ [high income]), smoking (current smoker/not current smoker), insulin use (yes/no), and BMI (normal $\left[<25 \mathrm{~kg} / \mathrm{m}^{2}\right]$, overweight [25-29.9 kg/m ${ }^{2}$, and obese $\left.\left[>30 \mathrm{~kg} / \mathrm{m}^{2}\right]\right)$ among adults aged 18 and over were used for this analysis.

Vital status and cause of death was ascertained through to the end of 2015 via linkage of the NHIS data to the NDI. Cancer deaths were classified according to underlying cause of death using International Classification of Diseases (ICD-9 [www.icd9data.com/ 2007/Volume1] for deaths prior to 1999, and ICD-10 [http://apps.who.int/classifications/icd10/browse/2016/en] for deaths occurring from 1999 onwards) (electronic supplementary material [ESM] Table 1). 
Statistical analyses To determine whether changes in cancer mortality rates over time differed in diabetes vs nondiabetes populations, we used weighted discrete Poisson regression to predict average marginal death rates according to baseline diabetes status and time period of follow-up (1988-1994, 1995-1999, 2000-2004, 2005-2009, 2010-2015). Because cohorts for each annual survey overlap during their follow-up time, we divided the continuous time-to-event survival data into discrete follow-up years. To reduce potential selection bias as a result of people with acute and life-threatening illness being less likely to be interviewed at baseline, we excluded all follow-up time of participants up to the end of the 2nd year of follow-up [5, 13]. We further limited the follow-up period to less than 10 years to reduce misclassification of respondents owing to incident diabetes during the follow-up period. We examined the effect of the time period exclusions with a sensitivity analysis which demonstrated that predicted mortality rates were substantially lower with the exclusions than without, which is consistent with the expected biases (see ESM Table 2).

Sample weights were adjusted for survey respondents who were excluded from analysis because of insufficient identifying data for NDI linkage [14]. In addition, weights in the discrete time-to-event data were calibrated to compensate for overlapping and ageing survey cohorts and to make the sample for each follow-up year nationally representative [15]. For results aggregated by time period, final weights were divided by the number of years in each time period [14].

Poisson regression models adjusted for time-varying age at follow-up, sex and race/ethnicity with robust variance estimation [15]. Hypothesis tests were conducted for differences by diabetes status, and for linear trends over time periods $\left(p_{\text {trend }}\right)$. We included an interaction effect between diabetes status and time period in the estimated rates based on significant periodby-diabetes status interactions. Annual cancer mortality rates, by diabetes status, were also stratified by sex, race/ethnicity, education and BMI. Site-specific cancer mortality rates were stratified by diabetes only. We used a $p$ value of $<0.05$ as an indication of statistical significance. We used SAS (version 9.4; https://www.sas.com/en_us/software/sas9.html; accessed 30 July 2019) and SUDAAN (https://www.rti.org/impact/sudaanstatistical-software-analyzing-correlated-data; accessed 30 July 2019) to account for the complex multistage sampling design and produce weighted estimates and contrasts, 95\% CIs and total case estimates. RRs and their $95 \%$ CIs were computed manually using the delta method [16].

Ethics NHIS is approved by the Research Ethics Review Board of the National Center for Health Statistics and the US Office of Management and Budget. All NHIS respondents provided oral consent prior to participation.

\section{Results}

Between 1988-1991 and 2007-2012, the total number of adults with reported diagnosed diabetes increased from 6.3 million to 20 million US adults. Among adults with diabetes, the proportion of men, proportion of those who were obese, proportion of Hispanics, proportion with a history of cancer, and proportion with newly diagnosed diabetes (diagnosed within 1 year) increased across time periods (Table 1). In contrast, the proportion of non-Hispanic white people, smokers, insulin users and less than high school educated adults decreased across time periods, while the proportion of adults with low income decreased between 1988-1991 and 2002-2006, and then increased. Similar patterns across time periods in adults without diabetes were observed, however, compared with adults without diabetes, those with diabetes were more likely to be older and non-Hispanic black, and to be obese and have lower levels of education.

Among adults with diabetes, age-adjusted cancer mortality rates (per 10,000 person-years) declined $25.5 \%$ from 39.1 (95\% CI 30.1, 50.8) in 1988-1994 to $29.7(26.6,33.1)$ in 2010-2015, $p_{\text {trend }}<0.001$ (Table 2). Among adults without diabetes, cancer mortality rates similarly declined $25.2 \%$ from $30.9(28.6,33.4)$ in $1988-1994$ to $23.2(22.1,24.2)$ in 2010 $15, p_{\text {trend }}<0.001$. The RR of cancer mortality remained approximately $30 \%$ higher in people with vs without diabetes, and this excess risk did not change over time (from RR 1.3 $[1.0,1.7]$ in 1988 - 1994 , to $1.3[1.1,1.4]$ in $2010-2015$ ).

By subgroup, in the population with diabetes, total cancer mortality rates declined significantly among men, nonHispanic white people, people educated to high school level, and overweight adults (Table 2). For all other subgroups, there were no statistically significant differences over time. Among the population without diabetes, statistically significant declines were noted among all subgroups, with the exception of Hispanic/other and non-Hispanic black adults, and adults with less than high school level education, where declines were noted, but linear trends were not significant. The RR between diabetic and non-diabetic populations remained similar over time in all subgroups, excluding adults who were obese, where it increased from 0.8 to 1.2 , respectively (Table 2).

By cancer type, for adults with and without diabetes, declines in multivariate adjusted mortality rates were observed for cancers of the lung, colorectal region, pancreas, breast and 'other' (Table 3). Between 1988-1994 and 2010-2015, the greatest relative declines among adults with diabetes were observed for pancreas $(55 \%)$ and breast $(65 \%)$ cancers, $p$ trend $<0.05$. The relative decline in lung cancer mortality rates was $27 \%$ for adults without diabetes, and a modest $7 \%$ decline for adults with diabetes. For melanoma, although similar relative declines were noted in both adults with and without diabetes, the decline was significant only for adults without 


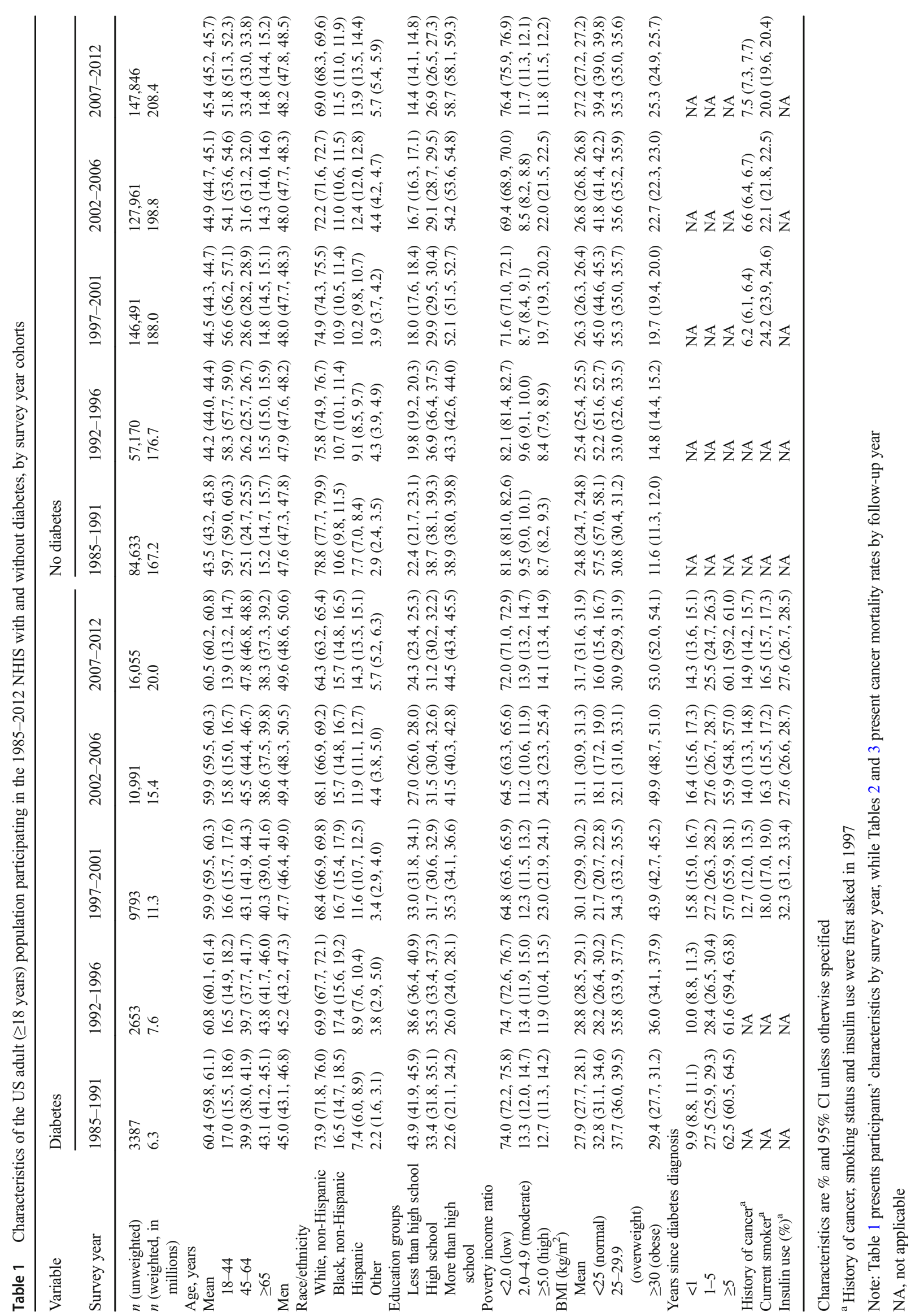


Table 2 Age-adjusted mortality rates from all cancers (per 10,000 person-years, 95\% CI), by period and diabetes status, stratified by sex, race/ethnicity, education level, poverty/income ratio and BMI status, USA 1988-2015

\begin{tabular}{|c|c|c|c|c|c|c|}
\hline Period & 1988-1994 & 1995-1999 & $2000-2004$ & 2005-2009 & 2010-2015 & $p_{\text {trend }}$ \\
\hline \multicolumn{7}{|l|}{ Total population } \\
\hline Diabetes & $39.1(30.1,50.8)$ & $37.4(30.2,46.4)$ & $38.3(32.3,45.4)$ & $33.0(29.4,37.1)$ & $29.7(26.6,33.1)$ & $<0.001$ \\
\hline No diabetes & $30.9(28.6,33.4)$ & $28.2(26.4,30.0)$ & $27.4(25.9,28.9)$ & $26.0(24.8,27.3)$ & $23.2(22.1,24.3)$ & $<0.001$ \\
\hline Excess risk (vs no diabetes) & $8.2(-2.3,18.7)$ & $9.3(1.1,17.5)$ & $10.9(4.2,17.6)$ & $7.0(3.0,11.0)$ & $6.5(3.1,9.9)$ & \\
\hline RR (vs no diabetes) & $1.3(1.0,1.7)$ & $1.3(1.1,1.7)$ & $1.4(1.2,1.7)$ & $1.3(1.1,1.4)$ & $1.3(1.1,1.4)$ & \\
\hline \multicolumn{7}{|l|}{ Sex } \\
\hline \multicolumn{7}{|l|}{ Men } \\
\hline Diabetes & $45.0(31.9,63.4)$ & $38.6(28.5,52.2)$ & $45.9(36.3,58.1)$ & $35.8(30.6,41.9)$ & $29.6(25.4,34.4)$ & 0.007 \\
\hline No diabetes & $35.3(31.7,39.4)$ & $33.0(30.3,35.9)$ & $32.9(30.5,35.6)$ & $29.2(27.4,31.1)$ & $25.3(23.6,27.0)$ & $<0.001$ \\
\hline Excess risk (vs no diabetes) & $9.7(-6.7,26.0)$ & $5.6(-6.2,17.4)$ & $13.0(1.8,24.2)$ & $6.6(0.9,12.3)$ & $4.3(, 0.5,9.1)$ & \\
\hline RR (vs no diabetes) & $1.3(0.9,1.8)$ & $1.2(0.9,1.6)$ & $1.4(1.1,1.8)$ & $1.2(1.0,1.5)$ & $1.2(1.0,1.4)$ & \\
\hline \multicolumn{7}{|l|}{ Women } \\
\hline Diabetes & $34.3(23.5,49.9)$ & $36.0(26.9,48.2)$ & $28.2(22.3,35.7)$ & $29.1(24.4,34.7)$ & $28.3(24.3,33.1)$ & 0.182 \\
\hline No diabetes & $27.4(24.3,30.9)$ & $24.0(21.9,26.3)$ & $22.6(20.9,24.5)$ & $23.1(21.7,24.6)$ & $21.2(19.8,22.7)$ & $<0.001$ \\
\hline Excess risk (vs no diabetes) & $6.9(-6.1,19.8)$ & $12.0(1.1,23.0)$ & $5.6(, 1.4,12.6)$ & $6.0(0.7,11.2)$ & $7.2(2.6,11.7)$ & \\
\hline RR (vs no diabetes) & $1.3(0.8,1.9)$ & $1.5(1.1,2.0)$ & $1.2(1.0,1.6)$ & $1.3(1.0,1.5)$ & $1.3(1.1,1.6)$ & \\
\hline \multicolumn{7}{|l|}{ Race/Ethnicity } \\
\hline \multicolumn{7}{|l|}{ White, non-Hispanic } \\
\hline Diabetes & $46.9(34.4,63.8)$ & $46.6(36.9,58.9)$ & $43.5(35.8,52.9)$ & $36.7(31.8,42.4)$ & $35.9(31.6,40.8)$ & 0.020 \\
\hline No diabetes & $33.5(30.8,36.5)$ & $31.5(29.2,33.8)$ & $29.6(27.8,31.5)$ & $28.7(27.3,30.2)$ & $24.7(23.4,26.1)$ & $<0.001$ \\
\hline Excess risk (vs no diabetes) & $13.3(-1.6,28.3)$ & $15.2(4.0,26.3)$ & $13.9(5.3,22.6)$ & $8.0(2.6,13.5)$ & $11.2(6.4,16.0)$ & \\
\hline RR (vs no diabetes) & $1.4(1.0,1.9)$ & $1.5(1.2,1.9)$ & $1.5(1.2,1.8)$ & $1.3(1.1,1.5)$ & $1.5(1.3,1.7)$ & \\
\hline \multicolumn{7}{|l|}{ Black, non-Hispanic } \\
\hline Diabetes & $30.2(16.1,56.6)$ & $20.7(12.4,34.7)$ & $32.2(22.5,46.1)$ & $33.0(25.2,43.2)$ & $20.2(15.7,26.0)$ & 0.472 \\
\hline No diabetes & $29.7(23.3,38)$ & $27.2(22.7,32.5)$ & $30.3(26,35.3)$ & $24.1(20.7,28.0)$ & $24.1(21.2,27.5)$ & 0.076 \\
\hline Excess risk (vs no diabetes) & $0.5(-19.4,20.4)$ & $-6.4(-18.6,5.7)$ & $1.9(-11.4,15.3)$ & $8.9(-1.0,18.8)$ & $-4.0(-10.0,2.1)$ & \\
\hline RR (vs no diabetes) & $1.0(0.5,2.0)$ & $0.8(0.4,1.3)$ & $1.1(0.7,1.6)$ & $1.4(1.0,1.9)$ & $0.8(0.6,1.1)$ & \\
\hline \multicolumn{7}{|l|}{ Hispanic/Other } \\
\hline Diabetes & S & $\mathrm{S}$ & $20.2(11.6,35.2)$ & $17.1(12.6,23.2)$ & $15.6(11.8,20.5)$ & 0.562 \\
\hline No diabetes & S & $\mathrm{S}$ & $15.3(12.8,18.4)$ & $15.4(13.4,17.8)$ & $15.4(13.5,17.6)$ & 0.436 \\
\hline Excess risk (vs no diabetes) & $\mathrm{S}$ & $\mathrm{S}$ & $4.9(-6.8,16.5)$ & $1.6(-3.8,7.1)$ & $0.1(-4.5,4.8)$ & \\
\hline RR (vs no diabetes) & S & S & $1.3(0.7,2.4)$ & $1.1(0.8,1.6)$ & $1.0(0.7,1.4)$ & \\
\hline \multicolumn{7}{|l|}{ Education level } \\
\hline \multicolumn{7}{|l|}{ Less than high school } \\
\hline Diabetes & $51.2(36.1,72.5)$ & $57.3(42.7,76.9)$ & $58.2(45.7,74.2)$ & $51.5(42.5,62.3)$ & $46.5(38.2,56.5)$ & 0.406 \\
\hline No diabetes & $51.8(46.4,57.8)$ & $45.3(41.1,50.0)$ & $46.3(42.1,51.1)$ & $46.6(42.9,50.6)$ & $44.0(40.0,48.3)$ & 0.058 \\
\hline Excess risk (vs no diabetes) & $-0.6(-19.9,18.7)$ & $12.0(-5.3,29.4)$ & $11.9(-3.1,26.8)$ & $4.9(-5.5,15.2)$ & $2.5(-7.3,12.3)$ & \\
\hline RR (vs no diabetes) & $1.0(0.7,1.4)$ & $1.3(0.9,1.7)$ & $1.3(1.0,1.6)$ & $1.1(0.9,1.4)$ & $1.1(0.9,1.3)$ & \\
\hline \multicolumn{7}{|l|}{ High school } \\
\hline Diabetes & $49.1(32.7,73.8)$ & $46.0(32.0,66.2)$ & $41.8(31.5,55.5)$ & $33.3(26.7,41.6)$ & $27.5(22.8,33.3)$ & 0.002 \\
\hline No diabetes & $30.2(26.4,34.6)$ & $28.5(25.7,31.7)$ & $28.4(25.9,31.2)$ & $27.4(25.3,29.6)$ & $22.9(20.9,25.1)$ & $<0.001$ \\
\hline Excess risk (vs no diabetes) & $18.9(-1.5,39.2)$ & $17.5(0.4,34.5)$ & $13.4(1.2,25.5)$ & $5.9(-1.8,13.6)$ & $4.6(-1.0,10.2)$ & \\
\hline RR (vs no diabetes) & $1.6(1.1,2.5)$ & $1.6(1.1,2.4)$ & $1.5(1.1,2.0)$ & $1.2(1.0,1.5)$ & $1.2(1.0,1.5)$ & \\
\hline \multicolumn{7}{|l|}{ More than high school } \\
\hline Diabetes & $29.7(18.6,47.3)$ & $17.7(10.5,29.8)$ & $25.8(18.2,36.3)$ & $24.2(19.7,29.8)$ & $23.4(19.4,28.1)$ & 0.787 \\
\hline No diabetes & $20.3(17.3,23.8)$ & $20.1(17.7,23.0)$ & $18.7(16.9,20.6)$ & $17.4(16.1,18.8)$ & $16.1(15.0,17.3)$ & $<0.001$ \\
\hline Excess risk (vs no diabetes) & $9.4(-4.8,23.6)$ & $-2.4(-12.0,7.1)$ & $7.1(-2.0,16.2)$ & $6.8(1.7,12.0)$ & $7.2(2.8,11.7)$ & \\
\hline RR (vs no diabetes) & $1.5(0.9,2.4)$ & $0.9(0.5,1.5)$ & $1.4(1.0,2.0)$ & $1.4(1.1,1.7)$ & $1.5(1.2,1.8)$ & \\
\hline
\end{tabular}


Table 2 (continued)

\begin{tabular}{|c|c|c|c|c|c|c|}
\hline Period & $1988-1994$ & 1995-1999 & $2000-2004$ & 2005-2009 & $2010-2015$ & $p_{\text {trend }}$ \\
\hline \multicolumn{7}{|l|}{ BMI status } \\
\hline \multicolumn{7}{|l|}{$<25 \mathrm{~kg} / \mathrm{m}^{2}$ (normal) } \\
\hline Diabetes & $31.6(21.5,46.3)$ & $41.5(27.9,61.7)$ & $33.0(23.2,47.0)$ & $34.0(26.2,44.1)$ & $26.1(20.1,33.8)$ & 0.272 \\
\hline No diabetes & $29.2(26.4,32.4)$ & $27.1(24.9,29.6)$ & $26.0(24.0,28.2)$ & $25.4(23.7,27.3)$ & $22.0(20.3,23.8)$ & $<0.001$ \\
\hline Excess risk (vs no diabetes) & $2.3(-10.2,14.9)$ & $14.3(-2.4,31.1)$ & $7.0(-4.8,18.8)$ & $8.6(-0.3,17.6)$ & $4.1(-2.8,11.0)$ & \\
\hline RR (vs no diabetes) & $1.1(0.7,1.6)$ & $1.5(1.0,2.3)$ & $1.3(0.9,1.8)$ & $1.3(1.0,1.8)$ & $1.2(0.9,1.6)$ & \\
\hline \multicolumn{7}{|l|}{$25-29.9 \mathrm{~kg} / \mathrm{m}^{2}$ (overweight) } \\
\hline Diabetes & $54.8(36.5,82.2)$ & $36.3(26.0,50.7)$ & $42.7(33.2,54.9)$ & $33.3(27.5,40.3)$ & $30.7(25.4,37.2)$ & 0.011 \\
\hline No diabetes & $32.6(28.6,37.2)$ & $30.9(27.8,34.3)$ & $29.1(26.6,32.0)$ & $28.0(25.9,30.3)$ & $24.6(22.8,26.6)$ & $<0.001$ \\
\hline Excess risk (vs no diabetes) & $22.1(-0.6,44.8)$ & $5.5(-7.1,18.1)$ & $13.5(2.3,24.8)$ & $5.2(-1.4,11.9)$ & $6.1(-0.1,12.2)$ & \\
\hline RR (vs no diabetes) & $1.7(1.1,2.6)$ & $1.2(0.8,1.7)$ & $1.5(1.1,1.9)$ & $1.2(1.0,1.5)$ & $1.2(1.0,1.5)$ & \\
\hline \multicolumn{7}{|l|}{$\geq 30 \mathrm{~kg} / \mathrm{m}^{2}$ (obesity) } \\
\hline Diabetes & $27.9(16.7,46.6)$ & $31.4(21.2,46.7)$ & $39.8(30.0,52.9)$ & $32.6(26.9,39.6)$ & $30.3(25.9,35.6)$ & 0.814 \\
\hline No diabetes & $34.3(27.9,42.2)$ & $25.9(21.9,30.6)$ & $28.5(24.9,32.8)$ & $24.3(21.9,27.0)$ & $24.6(22.2,27.2)$ & 0.006 \\
\hline Excess risk (vs no diabetes) & $-6.4(-21.9,9.0)$ & $5.5(-7.8,18.8)$ & $11.3(-0.6,23.2)$ & $8.3(1.7,14.9)$ & $5.7(0.3,11.2)$ & \\
\hline RR (vs no diabetes) & $0.8(0.5,1.4)$ & $1.2(0.8,1.9)$ & $1.4(1.0,1.9)$ & $1.3(1.1,1.7)$ & $1.2(1.0,1.5)$ & \\
\hline
\end{tabular}

S indicates suppressed cell (if $n<10$ )

Excess risk $=$ rate $($ diabetes $)-$ rate $($ no diabetes $) ; \mathrm{RR}=$ rate $($ diabetes $)-$ rate $($ no diabetes $) /$ rate $($ diabetes $)$

For unweighted counts of total cancer by subgroup, see ESM Table 3

diabetes, $p_{\text {trend }}<0.001$ due to the larger sample size. The RR in adults with vs without diabetes decreased for pancreatic, breast and 'all other' cancers, but did not marginally change for cancers of the lung or colorectal region or for melanoma (Table 3).

\section{Discussion}

We report several key findings in the trends in site-specific cancer mortality rates among US adults with and without diabetes. First, declines in cancer mortality rates were observed in both adults with and without diabetes. However, during the 2010-2015 time period, adults with diabetes remain approximately $30 \%$ more likely to die from cancer than people without diabetes. Furthermore, this RR has not improved over time. Second, rates of cancer mortality showed no significant change in some population subgroups, and the RR between adults with obesity, with and without diabetes, increased. Third, declines in total cancer mortality rates in adults with diabetes appear to be driven by large relative declines in cancers of the pancreas and breast, while for lung cancer, declines are modest.

In general, adults with diabetes have a $20-80 \%$ increased risk for cancer-specific mortality, depending on the cancer site [7]. Positive associations have been shown between baseline diabetes and risk of mortality from cancers of the colon, liver, pancreas and bladder [2, 17, 18], while associations for prostate, breast, endometrial and lung cancers are inconsistent $[2$,
17, 18]. Some evidence also supports an association between diabetes and risk of all-cause mortality in individuals with cancer $[19,20]$, but the evidence for cancer-specific mortality is varied [20-23]. Few studies, however, have examined rates of cancer-specific mortality over time, and none have previously examined trends in rates of site-specific cancers in adults with and without diabetes.

In the USA, Gregg et al. [5] reported similar modest declines in rates of total cancer mortality in adults with and without diabetes between 1988 and 2015, but did not examine trends in rates of site-specific cancer mortality. They showed that, while the proportion of deaths as a result of vascular diseases in adults with diabetes declined significantly over time (from $47.8 \%$ to $34.1 \%$ ), the proportion of deaths from cancer remained relatively stable, ranging from $15.9 \%$ to $19.9 \%$ [5]. In contrast, an Australian national diabetes registry reported an increase in the proportion of cancer deaths between 1997 and 2010, from $19 \%$ to $25 \%$ in women and from $25 \%$ to $34 \%$ in men with type 2 diabetes [24], but no change in age-standardised rates of cancer mortality [25]. However, when stratified by age group, declines in cancer mortality rates were noted in older age groups with type 2 diabetes, but a significant increase was observed among those aged $<40$ years [25]. Increases in rates of cancer mortality among young adults may be attributed to a rise in cancer incidence. In the USA, the risk of developing several obesity-related cancers has increased in younger adults with steeper increases observed in successively younger generations since the 1950s [26]. In the current study, a non-significant increase in rates 
Table 3 Trends in multivariate adjusted ${ }^{\mathrm{a}}$ cancer-specific mortality rates (deaths per 10,000 person-years, $95 \%$ CI), by period and diabetes status, USA 1988-2015

\begin{tabular}{|c|c|c|c|c|c|c|c|}
\hline Site-specific cancer & 1988-1994 & 1995-1999 & $2000-2004$ & 2005-2009 & 2010-2015 & $\begin{array}{l}\text { Relative } \\
\text { change }(\%)^{\mathrm{b}}\end{array}$ & $p_{\text {trend }}$ \\
\hline \multicolumn{8}{|l|}{ All cancers } \\
\hline Diabetes & $38.8(29.9,50.4)$ & $36.9(29.8,45.8)$ & $37.1(31.3,44.0)$ & $32.3(28.7,36.4)$ & $28.9(25.9,32.2)$ & 25.5 & $<0.001$ \\
\hline No diabetes & $31.0(28.7,33.5)$ & $28.2(26.5,30.0)$ & $27.5(26.0,29.1)$ & $26.1(24.9,27.3)$ & $23.2(22.2,24.4)$ & 25.2 & $<0.001$ \\
\hline Excess risk (vs no diabetes) & $7.8(6.7,8.8)$ & $8.7(7.9,9.5)$ & $9.6(9.0,10.3)$ & $6.2(5.8,6.6)$ & $5.6(5.3,6.0)$ & & \\
\hline RR (vs no diabetes) & $1.3(1.0,1.6)$ & $1.3(1.0,1.6)$ & $1.3(1.1,1.6)$ & $1.2(1.1,1.4)$ & $1.2(1.1,1.4)$ & & \\
\hline \multicolumn{8}{|l|}{ All cancers except prostate } \\
\hline Diabetes & $38.5(29.6,50.2)$ & $34.5(27.6,43.1)$ & $34.3(28.8,40.9)$ & $31.5(27.9,35.5)$ & $28.6(25.6,32.0)$ & 25.7 & $<0.001$ \\
\hline No diabetes & $29.1(26.9,31.5)$ & $26.4(24.8,28.2)$ & $25.9(24.4,27.4)$ & $24.6(23.5,25.8)$ & $22.0(21,23.1)$ & 24.4 & $<0.001$ \\
\hline Excess risk (vs no diabetes) & $9.4(8.3,10.4)$ & $8.1(7.3,8.8)$ & $8.4(7.8,9.1)$ & $6.8(6.5,7.2)$ & $6.5(6.2,6.9)$ & & \\
\hline RR (vs no diabetes) & $1.3(1.0,1.7)$ & $1.3(1.0,1.6)$ & $1.3(1.1,1.6)$ & $1.3(1.1,1.5)$ & $1.3(1.2,1.5)$ & & \\
\hline \multicolumn{8}{|l|}{ Lung } \\
\hline Diabetes & $8.5(5.1,14.2)$ & $11(7.3,16.7)$ & $11.2(8.2,15.4)$ & $8.2(6.4,10.5)$ & $7.9(6.5,9.6)$ & 7.1 & $<0.001$ \\
\hline No diabetes & $8.9(7.7,10.4)$ & $7.9(7.0,8.9)$ & $7.6(6.8,8.4)$ & $7.8(7.1,8.4)$ & $6.5(5.9,7.2)$ & 27.0 & $<0.001$ \\
\hline Excess risk (vs no diabetes) & $-0.5(-0.9,0.0)$ & $3.1(2.6,3.6)$ & $3.6(3.3,4.0)$ & $0.5(0.2,0.7)$ & $1.4(1.2,1.5)$ & & \\
\hline RR (vs no diabetes) & $1.0(0.6,1.6)$ & $1.4(0.9,2.1)$ & $1.5(1.1,2.1)$ & $1.1(0.8,1.4)$ & $1.2(1.0,1.5)$ & & \\
\hline \multicolumn{8}{|l|}{ Colorectal } \\
\hline Diabetes & $3.7(1.8,7.7)$ & $3.7(2.1,6.8)$ & $5.0(3.1,8.2)$ & $2.3(1.5,3.4)$ & $2.0(1.3,3.0)$ & 45.9 & 0.059 \\
\hline No diabetes & $3.7(2.9,4.7)$ & $2.9(2.4,3.4)$ & $2.7(2.3,3.3)$ & $2.2(1.9,2.6)$ & $2.0(1.7,2.4)$ & 45.9 & $<0.001$ \\
\hline Excess risk (vs no diabetes) & $0.1(-0.2,0.4)$ & $0.9(0.6,1.1)$ & $2.3(2.0,2.5)$ & $0.0(, 0.1,0.1)$ & $0.0(-0.1,0.1)$ & & \\
\hline RR (vs no diabetes) & $1.0(0.5,2.2)$ & $1.3(0.7,2.4)$ & $1.9(1.1,3.1)$ & $1.0(0.7,1.7)$ & $1.0(0.6,1.6)$ & & \\
\hline \multicolumn{8}{|l|}{ Pancreas } \\
\hline Diabetes & $4.9(2.5,9.5)$ & $2.9(1.4,5.8)$ & $1.5(0.7,3.5)$ & $3.0(2.0,4.3)$ & $2.2(1.5,3.2)$ & 55.1 & 0.028 \\
\hline No diabetes & $1.7(1.2,2.4)$ & $1.5(1.1,2.0)$ & $1.5(1.2,2.0)$ & $1.5(1.3,1.8)$ & $1.4(1.2,1.8)$ & 17.6 & $<0.001$ \\
\hline Excess risk (vs no diabetes) & $3.2(2.8,3.5)$ & $1.4(1.2,1.6)$ & $0.0(-0.1,0.1)$ & $1.5(1.4,1.6)$ & $0.8(0.7,0.8)$ & & \\
\hline RR (vs no diabetes) & $2.9(1.3,6.2)$ & $1.9(0.9,4.0)$ & $1.0(0.4,2.6)$ & $2.0(1.3,3.0)$ & $1.6(1.0,2.5)$ & & \\
\hline \multicolumn{8}{|l|}{ Melanoma } \\
\hline Diabetes & $5.5(3.2,9.5)$ & $3.6(2.0,6.6)$ & $2.1(1.3,3.5)$ & $4.4(3.2,6.2)$ & $4.2(3.0,6.0)$ & 23.6 & 0.117 \\
\hline No diabetes & $3.4(2.8,4.2)$ & $3.4(2.9,4.0)$ & $3.2(2.7,3.8)$ & $3.0(2.6,3.4)$ & $2.4(2.1,2.7)$ & 29.4 & $<0.001$ \\
\hline Excess risk (vs no diabetes) & $2.1(1.8,2.4)$ & $0.2(0.0,0.4)$ & $-1.1(-1.2,1.0)$ & $1.5(1.3,1.6)$ & $1.8(1.7,2.0)$ & & \\
\hline RR (vs no diabetes) & $1.6(0.9,2.9)$ & $1.1(0.6,2.0)$ & $0.7(0.4,1.1)$ & $1.5(1.0,2.1)$ & $1.8(1.2,2.6)$ & & \\
\hline \multicolumn{8}{|l|}{ Breast $^{\mathrm{c}}$} \\
\hline Diabetes & $10.1(4.8,21.5)$ & $4.4(2.1,8.9)$ & $4.3(2.3,7.9)$ & $4.4(2.9,6.6)$ & $3.5(2.5,5.0)$ & 65.3 & 0.047 \\
\hline No diabetes & $4.4(3.5,5.6)$ & $4.1(3.4,4.9)$ & $3.5(2.9,4.2)$ & $2.8(2.4,3.3)$ & $3.1(2.6,3.7)$ & 29.5 & $<0.001$ \\
\hline Excess risk (vs no diabetes) & $5.7(4.9,6.4)$ & $0.3(0.0,0.6)$ & $0.8(0.5,1.1)$ & $1.6(1.4,1.8)$ & $0.4(0.3,0.5)$ & & \\
\hline RR (vs no diabetes) & $2.3(1.0,5.1)$ & $1.1(0.5,2.2)$ & $1.2(0.7,2.3)$ & $1.6(1.0,2.4)$ & $1.1(0.8,1.7)$ & & \\
\hline \multicolumn{8}{|l|}{ All other cancers } \\
\hline Diabetes & $6.1(3.3,11.3)$ & $3.3(1.7,6.5)$ & $4.0(2.3,6.7)$ & $3.5(2.5,5.0)$ & $3.4(2.4,4.7)$ & 44.3 & 0.017 \\
\hline No diabetes & $3.7(2.9,4.8)$ & $3.3(2.8,4.0)$ & $3.2(2.8,3.7)$ & $3.4(3.0,3.8)$ & $2.8(2.5,3.2)$ & 24.3 & $<0.001$ \\
\hline Excess risk (vs no diabetes) & $2.4(2.0,2.8)$ & $0.0(-0.2,0.3)$ & $0.7(0.5,1.0)$ & $0.1(0.0,0.3)$ & $0.6(0.5,0.7)$ & & \\
\hline RR (vs no diabetes) & $1.6(0.8,3.2)$ & $1.0(0.5,2.0)$ & $1.3(0.7,2.2)$ & $1.0(0.7,1.5)$ & $1.2(0.8,1.8)$ & & \\
\hline
\end{tabular}

${ }^{\text {a }}$ Adjusted for age at follow-up time, sex, race/ethnicity

${ }^{\mathrm{b}}$ Relative change $=$ rate $(1988-1994)-$ rate $(2010-2015) /$ rate $(1988-1994) \times 100$

${ }^{\mathrm{c}}$ Women only, adjusted for age at follow-up time, race/ethnicity

Excess risk $=$ rate $($ diabetes $)-$ rate $($ no diabetes $) ; \mathrm{RR}=$ rate $($ diabetes $)-$ rate $($ no diabetes $) /$ rate $($ diabetes $)$

For unweighted counts of site-specific cancers, see ESM Table 4 
of cancer mortality was observed among adults who were both obese and had diabetes. Unfortunately, because of small numbers, we were unable to explore this trend by age group. However, given the high prevalence of both diabetes and obesity in the USA, and the increased number of years living with these conditions, there may be important implications for future cancer incidence and subsequent mortality, particularly among young adults [27].

Relative mortality reductions in total cancer, colorectal cancer and melanoma were similar in adults with and without diabetes. This finding may reflect populationwide reductions in exposure to risk factors such as smoking [28] and ultraviolet light [29], as well as the impact of national screening programmes [30] and therapeutic advances [31]. For pancreas and breast cancers, relative declines were greater for adults with than without diabetes. This may suggest more aggressive treatment among adults with diabetes, though this is, generally, not supported in the literature [32, 33]. Alternatively, use of diabetes medications, namely metformin, as well as the adoption of low fat diets may lead to reductions in cancer risk, though current evidence to support this is conflicting [34-37]. It may be more likely, for breast cancer, that adults with diabetes had a higher mortality rate from these cancers to begin with, and thus population-wide uptake of screening programmes may have caused greater absolute and relative reductions among adults with than without diabetes. In contrast, the relative reductions for lung cancer were considerably less for adults with than without diabetes. In the USA it has previously been reported that tobacco use between 1999 and 2010 declined in people without but not with diabetes [38]. It is therefore plausible that the smaller reduction in rates of lung cancer mortality may be due to a lack of decline in smoking rates in the diabetes population.

There are several mechanisms by which diabetes may increase the risk for cancer-specific mortality among people with cancer. There is considerable evidence that cancer patients with diabetes are treated less aggressively or receive modified anti-cancer treatment as compared with cancer patients without diabetes $[20,32,33]$. Studies also report that people with diabetes have a more advanced stage at cancer diagnosis [20, 33, 39], poorer response to treatment [40] and increased risk of treatment-related adverse effects [40-45] and short-term mortality [41, 45, 46] compared with cancer patients without diabetes. The current study is unable to differentiate the impact of diabetes on cancer incidence, vs treatment outcomes, on cancer-specific mortality.

This is the largest, nationally representative study to explore rates in site-specific cancer mortality rates over time in adults with vs without diabetes. Nonetheless, there are limitations to be considered. First, cause-of-death reporting is affected by medical opinion and subjective judgement that could shift over time or by the transition from ICD-9 to ICD-10. However, in a US study, the overall sensitivity between death certificate data and cancer registry diagnoses was $82.8 \%$ and $85.0 \%$ for ICD-10 and ICD-9, respectively, with confirmation rates $95 \%$ or greater for more commonly diagnosed cancers (e.g. prostate, breast and lung) [47]. We are not aware of research about the comparability of ICD reporting by diabetes status nor of studies that have validated cause-of-death reporting against adjudicated medical records over time to determine whether reporting is changing.

Second, diabetes was self-reported and changes in diabetes definitions could have affected the population with diagnosed diabetes, leading to detection of diabetes at earlier stages and lower mortality rates. In the current study, we showed an increase in the proportion of adults with diabetes with less than 1 year of disease duration. However, other national data show that there has been no change in the average age at diagnosis nationally [48]. In addition, both the content and structure of the NHIS changed in 1997. As such, the way information pertaining to diabetes was collected changed before and after this time. For example, between 1980 and 1996, only a subsample of participants (one of every six) was asked 'During the past 12 months, did you have diabetes?' From 1997 onwards, all sampled adults were asked 'Has a health professional ever told you that you have diabetes?' Third, all types of diabetes are included in the current analysis with the assumption that the vast majority $(\sim 90-95 \%)$ have type 2 diabetes [49]. Fourth, the NHIS does not include undiagnosed diabetes or incident cases of diabetes postbaseline and thus misclassification may have occurred for a small number of participants. Fifth, small case numbers may have led to an increased risk of a type I statistical error for several tests of linear trends among subgroups of the population. Last, our analyses did not adjust for multiple comparisons, and some of the trends in specific cancer deaths over time are based on fairly large CIs and warrant replication and continued monitoring.

Conclusions In the USA, between 1988-1994 and 2010-2015, rates of cancer mortality declined but remained approximately $30 \%$ higher in adults with vs without diabetes. While declines in rates were noted across most subgroups of the population, adults who were both obese and had diabetes did not experience a decline in cancer mortality rates over time. In addition, relative declines were not consistent across site-specific cancers, with considerable reductions in cancers of the pancreas and breast among adults with diabetes, but modest declines for lung cancer. This study highlights the continued need for greater cancer risk-factor mitigation in adults with diabetes to prevent premature death from cancer. 
Acknowledgements The authors thank the women and men who participated in the study, as well as all of the staff involved at the NCHS for the study design, data collection and data dissemination.

Data availability The data that support the findings of this study are available from the National Center for Health Statistics but restrictions apply to the availability of these data, which were used under licence for the current study, and so are not publicly available. Data are however available from the authors upon reasonable request and with permission of the National Center for Health Statistics.

Funding This research received no specific grant from any funding agency in the public, commercial or not-for-profit sectors.

Duality of interest The authors declare that there is no duality of interest associated with this manuscript.

Contribution statement JLH conducted the literature search, contributed to the conception and design of the study, interpreted the data and wrote the article. LJA contributed to the design of the study, performed the statistical analysis, assisted in drafting the article and revised the article for important intellectual content. YJC provided technical statistical support and revised the article for important intellectual content. KMB, EWG, HKW and NRB contributed to the design of the study and revised the manuscript for important intellectual content. GI conceived and designed the study and revised the manuscript for important intellectual content. JLH is the guarantor of this work and, as such, had full access to the all the data in the study and takes responsibility for the integrity of the data and the accuracy of the data analysis. All authors approved the final version of the manuscript.

\section{References}

1. Gregg EW, Zhuo X, Cheng YJ, Albright AL, Narayan KM, Thompson TJ (2014) Trends in lifetime risk and years of life lost due to diabetes in the USA, 1985-2011: a modelling study. Lancet Diabetes Endocrinol 2(11):867-874. https://doi.org/10.1016/ S2213-8587(14)70161-5

2. Rao Kondapally Seshasai S, Kaptoge S, Thompson A et al (2011) Emerging Risk Factors Collaboration. Diabetes mellitus, fasting glucose, and risk of cause-specific death. N Engl J Med 364(9): 829-841

3. Harding JL, Pavkov ME, Magliano DJ, Shaw JE, Gregg EW (2019) Global trends in diabetes complications: a review of current evidence. Diabetologia 62(1):3-16. https://doi.org/10.1007/s00125018-4711-2

4. Gregg EW, Hora I, Benoit SR (2019) Resurgence in diabetesrelated complications. JAMA 321(19):1867-1868. https://doi.org/ 10.1001/jama.2019.3471

5. Gregg EW, Cheng YJ, Srinivasan M et al (2018) Trends in causespecific mortality among adults with and without diagnosed diabetes in the USA: an epidemiological analysis of linked national survey and vital statistics data. Lancet 391(10138):2430-2440. https:// doi.org/10.1016/S0140-6736(18)30314-3

6. Vigneri P, Frasca F, Sciacca L, Pandini G, Vigneri R (2009) Diabetes and cancer. Endocr Relat Cancer 16(4):1103-1123. https://doi.org/10.1677/ERC-09-0087

7. Renehan AG, Yeh HC, Johnson JA, Wild SH, Gale EA, Moller H (2012) Diabetes and cancer (2): evaluating the impact of diabetes on mortality in patients with cancer. Diabetologia 55(6):1619-1632. https://doi.org/10.1007/s00125-012-2526-0
8. Harding JL, Shaw JE, Peeters A, Cartensen B, Magliano DJ (2015) Cancer risk among people with type 1 and type 2 diabetes: disentangling true associations, detection bias, and reverse causation. Diabetes Care 38(2):264-270. https://doi.org/10.2337/dc141996

9. Wild SH (2011) Diabetes, treatments for diabetes and their effect on cancer incidence and mortality: attempts to disentangle the web of associations. Diabetologia 54(7):1589-1592. https://doi.org/10. 1007/s00125-011-2169-6

10. Karlstad O, Starup-Linde J, Vestergaard P et al (2013) Use of insulin and insulin analogs and risk of cancer - systematic review and meta-analysis of observational studies. Curr Drug Saf 8(5):333348. https://doi.org/10.2174/15680266113136660067

11. National Center for Health Statistics. Office of Analysis and Epidemiology. The Linkage of National Center for Health Statistics Survey Data to the National Death Index - 2015 Linked Mortality File (LMF): methodology overview and analytic considerations, March 2019. Hyattsville, Maryland. Accessed 30 July 2019 from: www.cdc.gov/nchs/data/datalinkage/LMF2015 Methodology_Analytic_Considerations.pdf

12. National Center for Health Statisics. Centers for Disease Control and Prevention. About the National Health Interview Survey. Accessed 30 July 2019 from: www.cdc.gov/nchs/nhis/about_nhis. $\mathrm{htm} \#$ sample design

13. Griffiths RI, O’Malley CD, Herbert RJ, Danese MD (2013) Misclassification of incident conditions using claims data: impact of varying the period used to exclude pre-existing disease. BMC Med Res Methodol 13(1):32. https://doi.org/10.1186/1471-2288-13-32

14. National Center for Health Statistics, Office of Analysis and Epidemiology (2013) Use of survey weights for linked data files - preliminary guidance. Hyattsville, Maryland. Accessed 30 July 2019 from: www.cdc.gov/nchs/data/datalinkage/use of survey_weights_for_linked_data_files.pdf

15. Cheng YJ, Gregg EW, Rolka DB, Thompson TJ (2016) Using multi-year national survey cohorts for period estimates: an application of weighted discrete Poisson regression for assessing annual national mortality in US adults with and without diabetes, 2000 2006. Popul Health Metr 14(1):48. https://doi.org/10.1186/s12963016-0117-x

16. Long JS, Freese J (2001) Predicted probabilities for count models. Stata J 1(1):51-57. https://doi.org/10.1177/1536867X0100100103

17. Coughlin SS, Calle EE, Teras LR, Petrelli J, Thun MJ (2004) Diabetes mellitus as a predictor of cancer mortality in a large cohort of US adults. Am J Epidemiol 159(12):1160-1167. https://doi.org/ 10.1093/aje/kwh161

18. Lam EK, Batty GD, Huxley RR et al (2011) Associations of diabetes mellitus with site-specific cancer mortality in the Asia-Pacific region. Ann Oncol 22(3):730-738. https://doi.org/10.1093/annonc/ mdq405

19. Barone BB, Yeh HC, Snyder CF et al (2008) Long-term all-cause mortality in cancer patients with preexisting diabetes mellitus: a systematic review and meta-analysis. JAMA 300(23):2754-2764. https://doi.org/10.1001/jama.2008.824

20. van de Poll-Franse LV, Houterman S, Janssen-Heijnen ML, Dercksen MW, Coebergh JW, Haak HR (2007) Less aggressive treatment and worse overall survival in cancer patients with diabetes: a large population based analysis. Int J Cancer 120(9):19861992. https://doi.org/10.1002/ijc.22532

21. Peairs KS, Barone BB, Snyder CF et al (2011) Diabetes mellitus and breast cancer outcomes: a systematic review and meta-analysis. J Clin Oncol 29(1):40-46. https://doi.org/10.1200/JCO.2009.27. 3011

22. Snyder CF, Stein KB, Barone BB et al (2010) Does pre-existing diabetes affect prostate cancer prognosis? A systematic review. Prostate Cancer Prostatic Dis 13(1):58-64. https://doi.org/10. 1038/pcan.2009.39 
23. Stein KB, Snyder CF, Barone BB et al (2010) Colorectal cancer outcomes, recurrence, and complications in persons with and without diabetes mellitus: a systematic review and meta-analysis. Dig Dis Sci 55(7):1839-1851. https://doi.org/10.1007/s10620-0090944-8

24. Harding JL, Shaw JE, Peeters A, Guiver T, Davidson S, Magliano DJ (2014) Mortality trends among people with type 1 and type 2 diabetes in Australia: 1997-2010. Diabetes Care 37(9):2579-2586. https://doi.org/10.2337/dc14-0096

25. Harding JL, Shaw JE, Peeters A, Davidson S, Magliano DJ (2016) Age-specific trends from 2000-2011 in all-cause and cause-specific mortality in type 1 and type 2 diabetes: a cohort study of more than one million people. Diabetes Care 39(6):1018-1026. https://doi. org $/ 10.2337 / \mathrm{dc} 15-2308$

26. Sung H, Siegel RL, Rosenberg PS, Jemal A (2019) Emerging cancer trends among young adults in the USA: analysis of a population-based cancer registry. Lancet Public Health 4(3):e137e147. https://doi.org/10.1016/S2468-2667(18)30267-6

27. Landberg A, Falt A, Montgomery S, Sundqvist P, Fall K (2019) Overweight and obesity during adolescence increases the risk of renal cell carcinoma. Int J Cancer 145(5):1232-1237. https://doi. org/10.1002/ijc.32147

28. Imperatore G, Cadwell BL, Geiss L et al (2004) Thirty-year trends in cardiovascular risk factor levels among US adults with diabetes: National Health and Nutrition Examination Surveys, 1971-2000. Am J Epidemiol 160(6):531-539. https://doi.org/10.1093/aje/ kwh232

29. Watson M, Thomas CC, Massetti GM et al (2015) CDC grand rounds: prevention and control of skin cancer. MMWR Morb Mortal Wkly Rep 64(47):1312-1314. https://doi.org/10.15585/ mmwr.mm6447a2

30. Howlader N, Noone AK, Krapcho M, et al. (2019) SEER Cancer Statistics Review, 1975-2011 National Cancer Institute. Bethesda, MD, USA, 2014. Accessed 30 July 2019 from: https://seer.cancer. gov/archive/csr/1975_2011/

31. Pal SK, Miller MJ, Agarwal N et al (2019) Clinical Cancer Advances 2019: Annual Report on Progress Against Cancer From the American Society of Clinical Oncology. J Clin Oncol 37(10):834-849. https://doi.org/10.1200/JCO.18.02037

32. Chan JM, Latini DM, Cowan J, Duchane J, Carroll PR (2005) History of diabetes, clinical features of prostate cancer, and prostate cancer recurrence-data from CaPSURE (United States). Cancer Causes Control 16(7):789-797. https://doi.org/10.1007/s10552005-3301-z

33. Srokowski TP, Fang S, Hortobagyi GN, Giordano SH (2009) Impact of diabetes mellitus on complications and outcomes of adjuvant chemotherapy in older patients with breast cancer. J Clin Oncol 27(13):2170-2176. https://doi.org/10.1200/JCO.2008.17. 5935

34. Farmer RE, Ford D, Forbes HJ et al (2017) Metformin and cancer in type 2 diabetes: a systematic review and comprehensive bias evaluation. Int J Epidemiol 46(2):728-744. https://doi.org/10.1093/ije/ dyw275

35. Boyd NF, Stone J, Vogt KN, Connelly BS, Martin LJ, Minkin S (2003) Dietary fat and breast cancer risk revisited: a meta-analysis of the published literature. Br J Cancer 89(9):1672-1685. https:// doi.org/10.1038/sj.bjc. 6601314

36. Smith-Warner SA, Spiegelman D, Adami HO et al (2001) Types of dietary fat and breast cancer: a pooled analysis of cohort studies. Int
J Cancer 92(5):767-774. https://doi.org/10.1002/10970215(20010601)92:5<767::AID-IJC1247>3.0.CO;2-0

37. Thiebaut AC, Jiao L, Silverman DT et al (2009) Dietary fatty acids and pancreatic cancer in the NIH-AARP diet and health study. J Natl Cancer Inst 101(14):1001-1011. https://doi.org/10.1093/jnci/ djp168

38. Ali MK, Bullard KM, Saaddine JB, Cowie CC, Imperatore G, Gregg EW (2013) Achievement of goals in U.S. diabetes care, 1999-2010. N Engl J Med 368(17):1613-1624. https://doi.org/10. 1056/NEJMsa1213829

39. Fleming ST, Pursley HG, Newman B, Pavlov D, Chen K (2005) Comorbidity as a predictor of stage of illness for patients with breast cancer. Med Care 43(2):132-140. https://doi.org/10.1097/ 00005650-200502000-00006

40. Meyerhardt JA, Catalano PJ, Haller DG, Mayer RJ, Macdonald JS, Benson AB 3rd et al (2003) Impact of diabetes mellitus on outcomes in patients with colon cancer. J Clin Oncol 21(3):433-440. https://doi.org/10.1200/JCO.2003.07.125

41. Davila JA, Rabeneck L, Berger DH, El-Serag HB (2005) Postoperative 30-day mortality following surgical resection for colorectal cancer in veterans: changes in the right direction. Dig Dis Sci 50(9):1722-1728. https://doi.org/10.1007/s10620-005-2925-x

42. Freedland SJ, Banez LL, Sun LL, Fitzsimons NJ, Moul JW (2009) Obese men have higher-grade and larger tumors: an analysis of the Duke Prostate Center database. Prostate Cancer Prostatic Dis 12(3): 259-263. https://doi.org/10.1038/pcan.2009.11

43. Gross CP, McAvay GJ, Guo Z, Tinetti ME (2007) The impact of chronic illnesses on the use and effectiveness of adjuvant chemotherapy for colon cancer. Cancer 109(12):2410-2419. https://doi. org/10.1002/cncr.22726

44. Herold DM, Hanlon AL, Hanks GE (1999) Diabetes mellitus: a predictor for late radiation morbidity. Int J Radiat Oncol Biol Phys 43(3):475-479. https://doi.org/10.1016/S0360-3016(98) 00460-X

45. Little SA, Jarnagin WR, DeMatteo RP, Blumgart LH, Fong Y (2002) Diabetes is associated with increased perioperative mortality but equivalent long-term outcome after hepatic resection for colorectal cancer. J Gastrointest Surg 6(1):88-94. https://doi.org/10. 1016/S1091-255X(01)00019-1

46. Wilt TJ, Cowper DC, Gammack JK, Going DR, Nugent S, Borowsky SJ (1999) An evaluation of radical prostatectomy at Veterans Affairs Medical Centers: time trends and geographic variation in utilization and outcomes. Med Care 37(10):1046-1056. https://doi.org/10.1097/00005650-199910000-00008

47. German RR, Fink AK, Heron M et al (2011) The accuracy of cancer mortality statistics based on death certificates in the United States. Cancer Epidemiol 35(2):126-131. https://doi.org/10.1016/j.canep. 2010.09.005

48. Centers for Disease Control and Prevention. National Diabetes Surveillance System (2016) Available from https://www.cdc.gov/ diabetes/data/index.html. Accessed 30 July 2019

49. Centers for Disease Control and Prevention (2017) National Diabetes Statistics Report. Available from https://www.cdc.gov/ diabetes/data/statistics/statistics-report.html. Accessed 30 July 2019

Publisher's note Springer Nature remains neutral with regard to jurisdictional claims in published maps and institutional affiliations. 